\title{
The Prediction Research of Safety Stock Based on the Combinatorial Forecasting Model
}

\author{
Weiping Zhong, Lili Zhang \\ Department of Economics, Qinhuangdao Institute of Technology, Qinhuangdao, 066000, China
}

Keywords: Safety stock; Support vector machine (SVM); Radial basis function neural network (RBFNN); Genetic algorithm (GA) combination forecasting model

\begin{abstract}
The inventory is related to enterprise's production, sales and cash flow. Therefore, enterprises pay more and more attention to safety stock control. In this paper, on the basis of support vector machine (SVM) model and RBF neural network model, genetic algorithm (GA) combined forecasting model is established for enterprise safety stock prediction research. The example analysis results show that the predicted results of combined forecasting model is accurate, and it's a scientific, reasonable and effective method for safety stock predictive control.
\end{abstract}

\section{Introduction}

Since enterprise inventory is related to its cash flows, enterprises attach great importance to the problem of how to keep a safety inventory. Enterprises generally increase purchasing and production to meet the need of the production and sales, but the cost is the enterprise to take up more money. Therefore, the forecasting of enterprise safety stock can reduce the inventory cost, enhance the market competitiveness, and meet the customers' needs better, which has important practical significance.

In the long-term production and management practices, people summed up a variety of methods to determine the safety stock level, such as ABC classification, quantitative ordering method, marginal analysis, mathematical statistics methods and utility value method. With the development of computer technology and artificial intelligence, artificial intelligence is used for inventory management and safety stock forecasting by academic research. Among them, the neural network [1][2] is the most commonly used, and the BP neural network is the most popular[3][4]. Through the learning of prediction problem, we found that SVM and RBF neural network have a unique advantage in solving such problems. In order to improve the prediction accuracy, this paper will establish an optimized combination forecasting model based on these two methods, and use genetic algorithm to determine the weights.

\section{Safety stock prediction model}

\section{Support vector machine forecasting model}

Support vector machine (SVM) proposed by Vapnik et al, is based on VC dimension theory and structure risk minimum principle of statistical learning theory. According to the limited sample information, the model will find the best compromise between complexity and learning ability to get the best generalization ability[5].

Set (xi, yi) as a sample training set, where $\mathrm{xi}=\mathrm{Rn}$ is the input space, and yi is the output value. Construct the linear function:

$$
f(x)=w \varphi(x)+b
$$

Where, $\mathrm{w}$ is the input weight vector, and $\mathrm{b}$ is the threshold. Insensitive loss function $(\varepsilon)$ takes the following form:

$$
\left|y_{i}-f\left(x_{i}\right)\right|=\left\{\begin{array}{c}
0,\left|y_{i}-f\left(x_{i}\right)\right|<\varepsilon \\
\left|y_{i}-f\left(x_{i}\right)\right|-\varepsilon, \text { ohter }
\end{array}\right.
$$

Empirical risk function is as follows: 


$$
R_{\text {emp }}=\sum_{i=1}^{l}\left|y_{i}-f\left(x_{i}\right)\right|
$$

According to the structure risk minimum principle of statistical learning theory, support vector machine determine the regression function by minimizing the institutional risk function. So the SVM regression algorithm can be expressed as the following constrained optimization problem.

$$
\begin{aligned}
& \min \left(\frac{1}{2}\|w\|^{2}+C \sum_{i=1}^{l}\left|y_{i}-f\left(x_{i}\right)\right|\right) \\
& \text { s.t. }\left\{\begin{array}{l}
y_{i}-w \varphi(x)-b \leq \varepsilon+\xi \\
w \varphi(x)+b-y_{i} \leq \varepsilon+\xi^{*}
\end{array}\right.
\end{aligned}
$$

Where, $\xi$ and $\xi$ * respectively represent the upper and lower training error, and both of them are zero or greater.

Due to the high dimensionality feature space, it is almost impossible to solve the equation directly. But it can be turned into seeking the dual problem by using Wolfe dual techniques and introducing dot product kernel function $\mathrm{k}(\mathrm{xi}, \mathrm{xj})$.

$$
\begin{aligned}
& \max \left[\left(\alpha, \alpha^{*}\right)-\frac{1}{2} \sum_{i=1}^{l} \sum_{j=1}^{l}\left(\alpha, \alpha^{*}\right)^{T}\left(\alpha, \alpha^{*}\right)\left(x_{i}, x_{j}\right)+\sum_{i=1}^{l}\left(\alpha, \alpha^{*}\right) y_{i}-\sum_{i=1}^{l}\left(\alpha, \alpha^{*}\right) \varepsilon\right] \\
& \text { s.t. } \sum_{i=1}^{l}\left(\alpha, \alpha^{*}\right)=0,0 \leq \alpha, \alpha^{*} \leq C
\end{aligned}
$$

The regression estimate function is :

$$
f(x)=\sum_{i=1}^{l}\left(\alpha, \alpha^{*}\right) k\left(x_{i}, x\right)+b
$$

Set the inventory data $\mathrm{m}$ phases, and the impact indicators' number is $\mathrm{n} . \mathrm{X}=\{\mathrm{x} 1, \mathrm{x} 2, \cdots, \mathrm{xm}\}$ is the input set, and $\mathrm{Y}=\{\mathrm{y} 1, \mathrm{y} 2, \cdots, \mathrm{ym}\}$ is the output set.

\section{Radial basis function (RBF) neural network model}

Radial basis function network is a class of forward networks based on the function approximation theory, the learning of which is equivalent to find the best fit plane of training data in multidimensional space. Each hidden layer neuron transfer function of the network constitutes a basis function of fitting plane. Generally the Gaussian function is used, and its expression is:

$$
R_{i}(x)=\exp \left(-\frac{\left\|x-c_{i}\right\|^{2}}{2 \sigma_{i}^{2}}\right)
$$

$m$

(7)

Where $\mathrm{x}$ is an $\mathrm{n}$-dimension input vector, $\mathrm{c}$ is the $\mathrm{i}$-th basis function center, $\sigma_{\mathrm{i}}$ is the $\mathrm{i}$-th basis

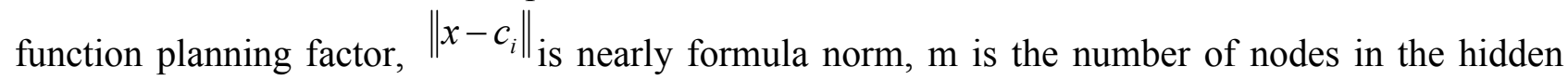
layer.

Set the input $X=(x 1, x 2, \cdots, x j, \cdots, x n)$, and the actual output $Y=(y 1, y 2, \cdots, y k, \cdots, y p)$. The input layer realizes the nonlinear mapping from $\mathrm{X}$ to $\mathrm{Ri}(\mathrm{x})$ The output layer realizes the linear mapping from $\mathrm{R}(\mathrm{X})$ to $\mathrm{yk}$. The kth output of the output layer' $\mathrm{s}$ neuron network is:

$$
\hat{y}_{k}=\sum_{i=1}^{m} w_{i k} R_{i}(x) \quad k=1,2, \cdots, p
$$

RBF network learning is the correction process of each network layer' $s$ weights and thresholds. This paper chooses newrb function to create an approximate radial basis network. 


\section{Combination forecasting model based on genetic algorithm}

Combined with support vector machine and RBF neural network model, the following combination forecasting model is set up:

$$
Y_{t}=\alpha * T_{t}+\beta * I_{t}+\varepsilon
$$

Where $\mathrm{Yt}$ is the predicted value, $\mathrm{Tt}$ is the prediction value of RBF neural network model, It is the prediction value of SVM model, $\mathrm{t}$ is the time serial number, $\alpha$ and $\beta$ are the weight factor, $\varepsilon$ represents the error value caused by other factors, which can be regarded as constant in a certain time.

The numerical constraints of $\alpha, \beta$ and $\varepsilon$ are as the following.

$$
\text { s.t. }\left\{\begin{array}{c}
\alpha+\beta \leq 1 \\
0<\alpha, \beta<1 ; \\
\varepsilon \geq 0
\end{array}\right.
$$

This paper will use genetic algorithm to solve the parameter values of $\alpha, \beta$ and $\varepsilon$. It is beneficial to optimize the combination forecasting model and avoid local optima.

\section{The empirical study of enterprise safety stock prediction}

This part selects AC company inventory data for empirical analysis. They are 20 groups of AC company' s most commonly used service parts' monthly minimum predefined actual history data[3].

\section{The influence factors of safety stock}

In AC company, the factors affecting raw material safety stock can be divided into internal factors and external combination factors.

Enterprise external comprehensive influencing factors are market demand, order delivery cycle, vendor products pass rate, storage costs, accessories ordering costs and stock losses. Safety stock is represented by $\mathrm{x} 1$. And these six influencing factors are respectively represented by $\mathrm{x} 2, \mathrm{x} 3, \mathrm{x} 4$, $x 5, x 6$ and $x 7 . X=\{x 2, x 3, x 4, x 5, x 6, x 7\}$ is the input set, and the safety stock is the output set.

\section{The original data}

Based on a monthly actual inventory data of AC company, set the six main factors as the input layer, and actual safety stock as the output layer. The original sample data are shown in Table 1. $\mathrm{x} 1$, $\mathrm{x} 2, \mathrm{x} 3, \mathrm{x} 4, \mathrm{x} 5, \mathrm{x} 6$ and $\mathrm{x} 7$ respectively represent safety stock, market demand, order delivery cycle, vendor products pass rate, storage costs, accessories ordering costs and stock losses. 
Tab.1 The original sample data

\begin{tabular}{|c|c|c|c|c|c|c|c|}
\hline NO. & $\begin{array}{l}\text { safety } \\
\text { stock }\end{array}$ & $\begin{array}{l}\text { market } \\
\text { demand }\end{array}$ & $\begin{array}{l}\text { order } \\
\text { delivery } \\
\text { cycle }\end{array}$ & $\begin{array}{c}\text { vendor } \\
\text { products pass } \\
\text { rate }\end{array}$ & $\begin{array}{l}\text { storage } \\
\text { costs }\end{array}$ & $\begin{array}{l}\text { accessories } \\
\text { ordering costs }\end{array}$ & $\begin{array}{l}\text { stock } \\
\text { losses }\end{array}$ \\
\hline 1 & 134 & 134 & 30 & 0.95 & 1 & 0.35 & 10 \\
\hline 2 & 504 & 432 & 35 & 0.91 & 1.5 & 0.25 & 5 \\
\hline 3 & 148 & 178 & 25 & 0.93 & 1 & 0.4 & 10 \\
\hline 4 & 22 & 67 & 10 & 0.95 & 2 & 0.5 & 15 \\
\hline 5 & 778 & 778 & 30 & 0.98 & 1 & 0.35 & 5 \\
\hline 6 & 82 & 123 & 20 & 0.95 & 1 & 0.4 & 15 \\
\hline 7 & 117 & 176 & 20 & 0.91 & 1.5 & 0.4 & 15 \\
\hline 8 & 165 & 330 & 15 & 1 & 1 & 0.35 & 15 \\
\hline 9 & 39 & 58 & 20 & 0.85 & 2 & 0.6 & 20 \\
\hline 10 & 519 & 623 & 25 & 0.95 & 0.5 & 0.35 & 10 \\
\hline 11 & 513 & 1025 & 15 & 0.96 & 0.5 & 0.3 & 5 \\
\hline 12 & 25 & 21 & 35 & 0.9 & 2.5 & 0.6 & 20 \\
\hline 13 & 547 & 821 & 20 & 0.99 & 1 & 0.3 & 5 \\
\hline 14 & 271 & 541 & 15 & 0.97 & 1 & 0.25 & 10 \\
\hline 15 & 485 & 970 & 15 & 0.99 & 1.5 & 0.2 & 5 \\
\hline 16 & 163 & 244 & 20 & 0.95 & 1 & 0.35 & 10 \\
\hline 17 & 68 & 51 & 40 & 0.93 & 2 & 0.5 & 20 \\
\hline 18 & 166 & 332 & 15 & 0.99 & 1.5 & 0.4 & 15 \\
\hline 19 & 59 & 88 & 20 & 0.98 & 1.5 & 0.5 & 20 \\
\hline 20 & 165 & 198 & 25 & 0.95 & 1 & 0.35 & 10 \\
\hline
\end{tabular}

Note: the above data is cited form the third part of the first document [3].

\section{Forecasting analysis of safety stock}

On the basis of the original data, the safety stock is forecasted respectively by SVM model, RBF neural network model and combination forecasting model based on genetic algorithm. The results are shown in Tab.2. And the comparison of safety stock prediction accuracy from number 2 to 20 are shown in figure 1. 
Tab.2 The safety stock prediction analysis of A C company

\begin{tabular}{|c|c|c|c|c|c|c|c|}
\hline \multirow{2}{*}{ No. } & \multirow{2}{*}{$\begin{array}{l}\text { Safety } \\
\text { stock }\end{array}$} & \multicolumn{2}{|c|}{ SVM model } & \multicolumn{2}{|c|}{ RBFNN model } & \multicolumn{2}{|c|}{ GA combination model } \\
\hline & & $\begin{array}{l}\text { Predicted } \\
\text { value }\end{array}$ & $\begin{array}{c}\text { Relative } \\
\text { error }(\%)\end{array}$ & $\begin{array}{l}\text { Predicted } \\
\text { value }\end{array}$ & $\begin{array}{c}\text { Relative } \\
\text { error }(\%)\end{array}$ & $\begin{array}{l}\text { Predicted } \\
\text { value }\end{array}$ & $\begin{array}{l}\text { Relative } \\
\text { error }(\%)\end{array}$ \\
\hline 2 & 504 & 503.68 & 0.06 & 503.75 & 0.05 & 504.13 & -0.03 \\
\hline 3 & 148 & 162.23 & -9.61 & 147.62 & 0.26 & 148.16 & -0.11 \\
\hline 4 & 22 & 22.55 & -2.51 & 22.02 & -0.09 & 22.41 & -1.85 \\
\hline 5 & 778 & 706.44 & 9.2 & 777.47 & 0.07 & 777.07 & 0.12 \\
\hline 6 & 82 & 81.69 & 0.37 & 81.82 & 0.22 & 82.2 & -0.24 \\
\hline 7 & 117 & 116.58 & 0.36 & 116.8 & 0.17 & 117.17 & -0.15 \\
\hline 8 & 165 & 164.47 & 0.32 & 164.69 & 0.19 & 165.07 & -0.04 \\
\hline 9 & 39 & 39.51 & -1.3 & 38.82 & 0.47 & 39.2 & -0.52 \\
\hline 10 & 519 & 496.33 & 4.37 & 519.07 & -0.01 & 519.2 & -0.04 \\
\hline 11 & 513 & 513.4 & -0.08 & 512.89 & 0.02 & 513.27 & -0.05 \\
\hline 12 & 25 & 25.41 & -1.65 & 24.97 & 0.11 & 25.36 & -1.43 \\
\hline 13 & 547 & 547.43 & -0.08 & 546.57 & 0.08 & 546.96 & 0.01 \\
\hline 14 & 271 & 272.02 & -0.38 & 270.36 & 0.24 & 270.76 & 0.09 \\
\hline 15 & 485 & 484.66 & 0.07 & 484.92 & 0.02 & 485.3 & -0.06 \\
\hline 16 & 163 & 162.45 & 0.34 & 162.76 & 0.15 & 163.13 & -0.08 \\
\hline 17 & 68 & 68.41 & -0.61 & 67.79 & 0.3 & 68.18 & -0.27 \\
\hline 18 & 166 & 165.5 & 0.3 & 165.34 & 0.4 & 165.72 & 0.17 \\
\hline 19 & 59 & 59.25 & -0.43 & 58.57 & 0.74 & 58.95 & 0.08 \\
\hline 20 & 165 & 176.29 & -6.84 & 161.46 & 2.14 & 162.01 & 1.81 \\
\hline
\end{tabular}

(1) The results analysis of single forecasting model

Calculating the mean square error (MSE) of two individual forecasting models and combination forecasting model with the actual value, we get that the MSE of SVM model is 17.72, the MSE of RBF neural network model is 0.88 . It can be learned that the fitness of the RBF neural network model with the actual value is higher, which means the RBF neural network model having a higher accuracy.

From the data table, we can see that both the predictions of SVM model and RBF neural network model are very close to the actual value. In the SVM model, in addition to the number3, number 5, and number 20 , the relative error of prediction value and actual value are small and below $5 \%$. Since the RBF neural network model has a higher forecasting accuracy, the relative errors are all below $3 \%$.

The trend curve in the figure shows that RBF neural network predictive value is closer to the actual value. Comparatively speaking, the curve of SVM model's predictive value is smooth, and can not show prominent changes in individual years. 




Fig.1 The comparison of safety stock prediction accuracy from number 2 to 20

(2) The result analysis of combination forecasting model

The mean square error of combination forecasting model with actual value is 0.74 . The result of combination forecasting model is better than the individual prediction. The relative error of combination prediction value and actual value are all below $2 \%$, which shows the high accuracy of combination forecasting model. As can be seen from figure 2, the combination predictive value and the actual value has a higher inosculation, it combines the advantages of the two single prediction, and the trend is closer to the actual value.

The above analysis shows that the two individual forecasting models' predictions are close to the actual values, and the combination forecasting model has a higher accuracy. The results reflect the science and rationality of the combination forecasting, and it can be used to promote research in various academic fields.

\section{Summary}

Since inventory has a important influence on the flow funds, companies pay more attention to the safety stock. The prediction of enterprise' $\mathrm{s}$ safety stock is a gray complex nonlinear system affected by a variety of factors. In this paper, the combination forecasting model is built based on the SVM model and RBF neural network model, and the weights are determined by genetic algorithm. The example analysis shows that the results of combination forecasting model have high accuracy. And it is a scientific, reasonable, and effective method for safety stock forecasting.

\section{References}

[1] $\mathrm{Gu}$ Bing. The safety stock forecasting of manufacturing enterprise based on BP neural network .Technology and Enterprise, 2013(19):107-107.

[2] Xi Ning. The application of neural network in the company's safety stock management. Fujian Computer, 2011,27(4): 143-144.

[3] Song Zhihao, Zheng Yongqian. The safety stock optimization analysis of company AC based on BP neural network .Market Modernization, 2013(16): 69-71.

[4] Zhao Yunfeng, Liu Wanjun. The application of artificial neural network in the ERP system. Computer Application, 2005, 25(4):748-750. 
[5] Shi Feng, Wang Xiao Chuan, Yu Lei, et al. The MATLAB neural network analysis of 30 cases. Beijing: Beijing University of Aeronautics and Astronautics Press, 2010.

[6] Ge Hong, Mao Zong-yuan. The Analysis of t he Local Search Efficiency of Genetic Neural Networks and the Improvement of Algorithm [A]. Processing of $t$ he $4 \mathrm{t} h$ World Congress on Intelligent Cont rol and Automation[ C] . Hefei : Press of East China University of Science and Technolgy ,2002.

[7] Fogel D B. Asymptotic Convergence Properties of Genetic Algorithms and Evolutionary Programming : Analysis and Experiments[J] .Cybernetics and System ,1994,25 (6) :389 - 407.

[8] Gambardella L M , Dorigo M. Solving symmetric and asymmetric TSPs by ant colonies[A]. In Proceedings of $t$ he IEEE international conference on evolutionary computation ( ICEC '96) [C]. USA : IEEE Press , 1996. 622 - 627. 